vorhanden sein sollten, ist woll nicht der Fall, denn man findet an vielen Orten Mittelformen zwischen Aspidium lobatum Sw. und aculeatum Sw., obgleich weit und breit das Aspidiun aculeatum Sw. nicht zu finden ist." Diese Bemerkung spricht jedoch nur für die Annahme Luerssen's, dass Aspidium lobatum Sw. mit Aspidium aculeatum Sw. als Formen einer Art zu vereinigen sind, während Aspidium Braunii Spenn. als eigene Art von diesen zu trennen ist, denn nirgends wird angeführt, dass an einem Standorte „Uebergangsformen" von Aspidium lobatum Sw. und Aspidium aculeatum Sw. zu Aspidium Braunii Spenn. beobacbtet worden wären, an dem typisches Aspidium Braunii Spenn. gefehlt hätte; keineswegs aber spricht diese Bemerkung gegen die Annahme des Bastardes Aspidium lobatum Sw. $\times$ Braunii Spenn.

In seinen späterën Abbandlungen und Werken bleibt Milde seiner Meinung treu und spricht nur von "Uebergangsformen". (Die schlesischen Gefäss-Kryptogamen in Oest. bot. Wochenblatt IX. 1859 S. 53; Die höh. Sporenpfl. Deutschl. u. d. Schweiz 1865 S. 66; Filices Europae et Atlantidis 1867 p. 109, 110.)

E. Fiek (Flora von Schlesien 1881 S. 553) gibt zu Aspidizum Braunii Spenn. folgende Note: „An der Eule, jm Gesenke z. B. um Gräfenberg und an der Czantory bei Ustron finden sich dentliche "Zwischenformen zur Var. a der vorigen Art (=lobatum Sw.). die zuerst von Milde, vielleicht mit Recht, als Bastarde (Wo?), dann aber als nieht hybride Uebergänge angesehen wurden."

(Fortsetzung folgt.)

\title{
Eine neue Sambucus-Art aus dem Himalaya.
}

\author{
Von Dr. Richard R. v. Wettstein (Wien).
}

(Mit Tafel II und 3 Holzsclinitten.)

Sambucus Gautschii Wettstein in Herb. mus. botan. univ. Vindob. 1889.

Herbacea, rhizomate repente, perennante, cylindrico, internodiis 8-15 cm. longis, lignoso. Caules erecti, $70-120 \mathrm{~cm}$. longi, simplices vel putati parce ramosi, glaberrimi, virides, in parte inferiore teretiusculi, superiore 8-16- furcati, $1-1^{1 / 2} \mathrm{~cm}$. crassi, demum modice lignosi, intus medullosi. Folia opposita, laete viridia, petiolata, impari-pinnato-composita, 3-4juga, cum petiolo $20-25 \mathrm{~cm}$. longa, supra glaberrima nitida, subtus glabra vel minutissime glandulosa, foliolis ovato-lanceolatis, apicem versus attenuatis, inferioribus sessilibus vel breviter petiolatis, superioribus parum decurrentibus, omnibus $8-14 \mathrm{~cm}$. longis, $25-4 \mathrm{~cm}$. latis, acute serratis, serris apicem versus vergentibus. Petiolus communis supra canaliculatus, infra rotundatus. Stipulae foliaceae, ovatae, serratae. 
Ad basin folioli cuinsque foliorum superiorum nectarium extranuptiale, foliis junioribus solum secernans, situm est. Flores parvi, ca. $3-4 \mathrm{~mm}$. diametro in cymis compositis multifloris, latis, applanatis, circa $10 \mathrm{~cm}$. diametro, ramis primariis quinatis, pedunculis tenuibus glabris. Calyx tubo striato obovato, laciniis acutis viridibus glabriusculis corollam ante anthesin superantibus. Corolla laciniis quinque ovatis, obtusiusculis concavis, albo-lutescentibus, staminibus a,equilongis. Stamina 5, antheris extrorsis luteis; patentia. Inflorescentia fructifera erecta, baccis erectis, ovatis, immaturis rubentibus maturis nigris vix carnosis.

Plantia tota odore ei Sambuci Ebuli L. simili. Fructus ab avibus eduntur.

Syn. Sambucus Ebulus Hooker f. et Thomson in Journ. of the proc. of the Linn. Soc. II, p. 179 (1858). - Clarke in Hooker Flora of Brit. Ind. III, p. 2 (1882). - Non Linné.

Patria: India orientalis. Provincia Kashmir: in montibus Himalayae editioribus in altitudine pedum angl. 6000-10.000 frequens. Specimina descripta culta in horto botanico Vindobonensi ann. $1886-1890$.

Speciem hanc denominavi in honorem principis ministri Dr. P. Gautsch Baro de Frankenthurn, cujus cura hortus botanicus universitatis Vindobonensis non solum a periculis ei minantibus liberatus et in futurum munitus est, sed etiam institutionibus ad usum scientiae praeclarissimis instruitur.

Die Samen der in den vorstehenden Zeilen beschriebenen Sambucus-Art erhielt der botanische Garten der Wiener Universität aus dem botanischen Garten in Shahjahanpur im Jahre 1886 durch Vermittlung des k. k. Handelsmuseums in Wien. Ans diesen Samen wurden fünf Pflanzen herangezogen, die im Jahre 1889 zum ersten Male blühten und Früchte reiften. Im Winter nur leicht gedeckt, erwies sich die Pflanze als vollkommen winterhart. Die einjährigen Sprosse beginnen in der zweiten Hälfte des Monates April (im Jahre 1890 am 13. April) auszutreiben, die Blüthen öfinen sich Anfang Juni, die ersten Früchte reifen Anfang September.

Die Pflanze ist eine entschieden krautige, wenngleich der Stengel, ebenso wie jener von Sambucus Ebulus L. bis zum Herbste einen relativ starken Holzcylinder entwickelt. Die Innovationsverhältnisse sind jenen der genannten Art ähnlich und insofern von Interesse, als die Analogie des Rhizoms mit den Langtrieben der strauchigen Sambucus-Arten deutlich hervortritt. Das in einem Jahre gebildete, ausläuferartige Rhizomstïck (Fig. 1) besteht aus mehreren (2-7) Internodien. An jedem Knoten sind zwei seitliche Knospen $(K)$ angelegt, während der ganze Spross in eine End- $\left(K_{I}\right)$ und zwei Seitenknospen endet. Im nächsten Jahre (Fig. 2) entwickelt sich zunächst die Endknospe zum oberirdischen Spross, während sich das Rhizom aus einer am unteren Ende dieses oberirdischen Sprosses anftretenden Knospe $\left(K_{\mathrm{II}}\right)$ fortsetzt. Regelmässig im dritten Jahre, abnormerweise 
bei Verletzung des ersten oberirdischen Sprosses oder bei besonders üppigem Wachsthum schon früher, entwickeln sich die an den Knoten des im ersten Jahre gebildeten Rhizomstückes stehenden Knospen zu oberirdischen Sprossen (Fig. 3). Diese Sprosse werden zugleich zn Ausgangspunkten neuer Rhizomäste, welche nach Zugrundegehen des alten Rhizomstückes zu selbstständigen Pfanzon werden können.

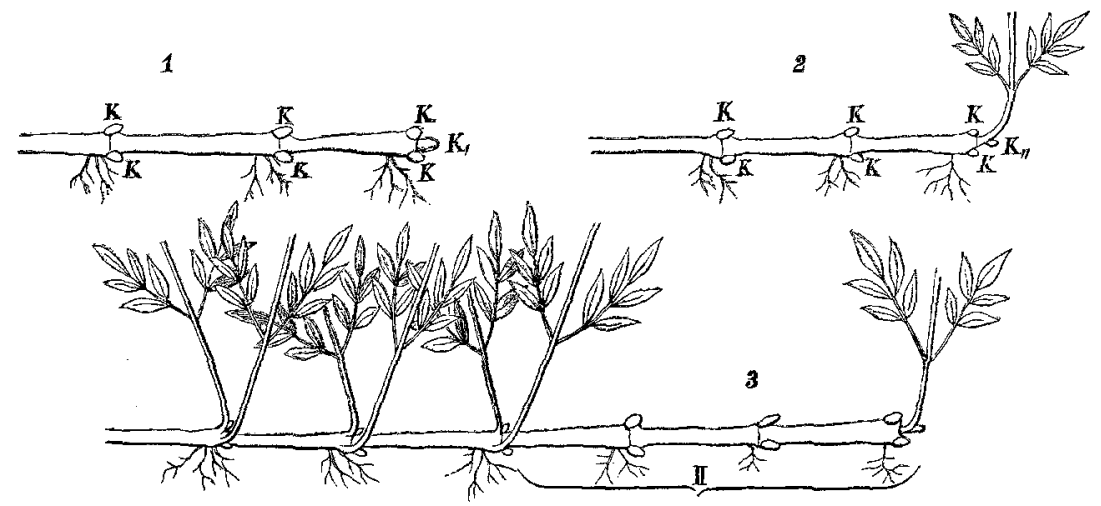

Schematische Darstellung der Sprossfolge. - Fig. 1. Rhizomstack, am Ende des 1. Vegetationsjahres gebildet. $K$ Knospen. - Fig. 2. Dasselbe Rhizomstück im Frahjahre des 2. Vegetationsjahres. - Fig. 3. Dasselbe Rhizomstack im Frthlinge des 3. Vegetationsjahres. II. ist das aus der Knospe $K_{I}$ (Fig. 2) hervorgegangene Rhizomstuek.

In systematischer Hinsicht stellt Sambucus Gautschii durch mehrere Eigenthümlickeiten eine Verbindung zwischen dem krautigen, bisher isolirt stehenden S. Ebulus L. und den strauchartigen Sambucus-Arten her. Dem ersteren nähert er sich durch die einjährigen unverzweigten Stengel und die Blattform, mit einigen der letzteren hat er die Gestalt des Blüthenstandes und die Form der Blüthentheile gemein.

Von S. Ebulus L. unterscheidet sich die neue Art durch die durehschnittlich geringere Zahl der Blättchenpaare, durch die breitere Gestalt und die eigenthümliche Zahnung der Theilblättchen, durch die fünfstrahlige Inflorescenz, die weitans kloineren Blüthen, lang zugespitzten Kelchzipfel, die stumpfen, concaven Corollblätter, die nicht aufrecht stebenden Staubgefässe mit gelben Antheren und kleineren Beeren. Weniger vou Bedeutung ist, dass die Blätter von $\boldsymbol{S}$. Gautschii unterseits ganz kahl oder schwach papillös-drüsig sind, dass seine Blattstiele an der Unterseite abgerundet sind und dass schliesslich überhaupt die Farbe der ganzen Pflanze viel heller als jene von Samb. Ebulus ist. Im botanischen Garten wurden beide Pflanzen nuter ganz gleichen Verhältnissen cultivirt, trotzdem war S. Gautschï in der Entwickelung dem S. Ebulus stets durchschnittlich um etwa 10 Tage voraus, wie ans der nachstehenden kleinen Tabelle entnommen werden kann. 


\begin{tabular}{|l|c|c|}
\hline & S. Gautwchii & S. Ebulus \\
\hline Erstes Blatt sichtbar & 16. April & 25. Apri1 $\left.{ }^{1}\right)$ \\
\hline Erste Blüthe geöffnet. & 2. Juni & 12. Juni ${ }^{2}$ ) \\
\hline
\end{tabular}

Wenn ich die strauchigen Sambucus-Arten gleichfalls in einen Vergleich mit $\boldsymbol{S}$. Gautschii ziehe, so geschieht dies blos, weil die B]üthenstände und Blätter einzelner eine gewisse Aehnljchkeit zeigen, dje bei Benützung von Herbarmateriale immerhin zu Verwechslungen führen könnte.

In erster Linie ist in dieser Hinsicht $S$. adnata Wallich (De Candolle Prodrom. IV. p. 322. (1830) zu beachten, der aber an den verzweigten Aesten, den unterseits behaarten Blättern, und der im unteren Theile beblätterten, 3 strahligen Inflorescenz leicht zu erkennen ist. - S. Javanica Reinw. (in Blume Bijdr. ned. Lud. p. 657) und $\boldsymbol{S}$. Chinensis Lindl. (Transact. Soc. hort. Lond. VI. p. 297) haben am Grunde beblätterte Inflorescenzen, kugelige Früchte und eine wesentlich andere Berandung der Blätter. - Mit S. nigra Linné (Spec. plant. ed. 1. p. 269 (1753) hat S. Gautschii nur in dem flach ausgebreiteten, vielstrahligen kleinblüthigen Blüthenstande eine Aehnlichkeit und anch diese schwindet bei eingehender Betrachtung, da die Blüthen der ersteren grösser sind, dabei stumpfere, rein weisse Corollblätter tragen (vgl. Taf. II, Fig. 6 u. 7).

Trotz der zahlreichen und auffallenden Unterschiede zwischen Sambucus Gautsch $\ddot{~} i$ und S. Ebulus sind bejde doch verwechselt worden. S. Gautschii ist nämlich dieselbe Pflanze, welche Thoms on und Hooker fil. in der Abhandlung „Praecursores ad floram Indiam" (Journ. of the proc. of the Linn. Soc. II. p. 179) als $S$. Ebulus L. bezeichneten. Ich überzeugte mich davon durch ein im Herbare des k. k. Hofmuseums in Wien aufbewahrtes Exemplar, welches von den Genannten gesammelt wurde und die Etiquette trägt "Sambucus Ebulus L. ? Hab. Himal. bor. occ. - Kishtwar 6-9000*. "Herb. Ind.-Or. Hook. fil. et Thomson." Der angegebene Standort ist der von den Autoren auch a. a. 0 . verzejchnete und dio Pflanze stimmt mit den mir vorliegenden Exemplare des S. Gautschii vollkommen überein.

Auf die Angabe Hooker's und Thomson's hin hat Clarke in Hooker's Flora of Brit. India III. p. 2. (1882) Sambucus Ebulus L. für Ost-Indien angegeben. Da er sich aber dabei blos auf obige Angahe stützt und ausdrücklich bemerkt, dass die Exemplare aus Kashmir reichstrahlige und grössere Inflorescenzen aufweisen ("Corymb ... usually larger and many-rayed in Kashmir specimens"), so ist als sicher anzunehmen, dass $S$. Ebulus L. im Himalaya ganz fohlt und daselbst durch $S$. Gautschii vertreten wird. Mit Rücksicht darauf erschien es mir von Wichtigkeit, die Ver-

) $1890 .-2) 1889$. 
breitungsgebiete der beiden Arten genau zu bestimmen, insbesondere zu eruiren, ob S. Gautschii nicht weiter nach Westen vorkomme, d. h. ob nicht eine der Angaben über das Vorkommen von S. Ebulus im westlichen Asien sich gleichfalls auf jene Art beziehe.

Auf Grund eines reichen Herbarmateriales kann ich constatiren, dass Sambucus Ebulus L. entsprechend den üblichen Angaben ${ }^{1}$ ) vom mittleren Schweden und pördlichen Russland über ganz Europa bis nach Nordafrika und Westasien verbreitet ist. Die östlichsten Standorte, von denen ich die Pflanze sah, sind: Diabekir in Mesopota-

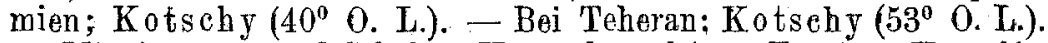
- Mt. Aoroman und Schahu; Haussknecht. - Taurien; Karelin (ca. $45^{0}$ o. L.).

S. Gautschii ist dagegen bisher blos aus dem westlichen Himalaya (ca. $75-80^{\circ}$ o. L.) bekannt geworden.

Schliesslich möchte jch nicht unerwähnt lassen, dass, nachdem ich im rergangenen Jabre S. Gautschii untersucht und im Herbare des botanischen Museums der k. k. Universität als neue Art aufgestellt hatte, Herr Dr. Karl Fritsch unabhängig von meinen Arbeiten gelegentlich einer Revision der Sambucus-Àrten des Herbariums des $\mathrm{k} . \mathrm{k}$. naturhistorischen Hofmuseums, gleichfalls das aus dem Himalaya unter dem Namen S. Ebulus L. vorliegende Exemplar als einer anderen Art angehörig erkannte und diese anf der Herbaretiquette $S$. Thomsoni nannte.

\section{Tafel-Erklärung.}

Fig. 1. Blühender Spross von \$. Gautschii Wettst. rerkl.

- 2 u. 3. Blüthen desselben, vierfach vergrössert, - Fig. 1-3 nach der Natur u. zw. nach Exemplaren des Wiener botan. Gartens.

Fig. 4 u. 5. Blüthen von $S$. Ebulus L. vierfach vergr.

-6 u. $7 . \quad-\quad-$ S. nigra L. vierfach vergr.

\section{Litteratur-Uebersicht. $\left.{ }^{2}\right)$}

April 1890.

Allescher A. Ueber einige aus dem südlichen Dentschland weníger bekannte Sphaeropsideen und Melanconieen. (Bot. Centralbl. 1890.) $8^{0} .9 \mathrm{~S}$.

Zusätze zum III. Bd, von Saccardo's Sylloge. Nene Arten : Actinonema Lonicesae alpigenae, A. Fraxini. A. Tiliae, A. Ulmi, A. Polagrariae, A. Pyrolae, Septoria Lonicerae, Pestalozzia Sarothamni, P. Comi.

1) Vergl. z. B. Nyman Conspect. flor. Europ. p. 321. - Boissiex Flora Orient. III. p. 2.

$\left.{ }^{2}\right)$ Die "Litteratur-Uebersicht" strebt Vollständigkeit nur mit Rücksicht auf jene Abhandlungen an, die entweder in Oesterreich-Ungarn erseheinen oder sich anf die Flora dieses Gebietes direct oder indirect beziehen, ferner auf selbstständige Werke des Auslandes. Zur Eraielang thunlichster Vollständjgkeit werden die Herren Autoren und Verleger um Einsendung von neu erschienenen Arbeiten höflichst ersucht.

Die Red. 